\title{
A single center's experience with noninvasive prenatal testing
}

\author{
Carmen J. Beamon, MD, MPH${ }^{1}$, Emily E. Hardisty, MS, CGC' ${ }^{1}$ Sarah C. Harris, MS, CGC ${ }^{2}$ \\ and Neeta L. Vora, MD ${ }^{1}$
}

Purpose: Massively parallel sequencing to detect fetal aneuploidy has high sensitivity and specificity for the detection of trisomies 21 , 18 , and 13 in high-risk populations. The purpose of our study was to review our institution's experience with the use of noninvasive prenatal testing for aneuploidy screening.

Methods: This was a descriptive study of patients who had undergone noninvasive prenatal testing between January and September 2012 at the UNC Prenatal Diagnosis unit.

Results: Two hundred and eight women had undergone noninvasive prenatal testing during the study period. The majority of patients were white $(62.9 \%)$ and of advanced maternal age (71.2\%). The fetal fraction was below the threshold in three obese patients (1.4\%). An abnormal noninvasive prenatal test (aneuploidy detected or "unclassified" result) was reported in 6.3\% (13/208) of the patients.

\section{INTRODUCTION}

Prenatal screening for fetal aneuploidy is routinely offered to all pregnant women. ${ }^{1,2}$ The options for screening have previously been limited to the use of markers in maternal serum and fetal anatomical ultrasound, with sensitivities of $69-95 \%$ for the detection of trisomies 21,18, and 13. ${ }^{1}$ Diagnostic testing via chorionic villus sampling (CVS) and amniocentesis is available for the detection of chromosomal abnormalities, but uptake is limited by the risk of pregnancy loss. As the percentage of women of advanced maternal age steadily increases, more sensitive screening tests with lower false-positive rates are needed. ${ }^{3}$

Owing to advances in massively parallel sequencing of maternal plasma, noninvasive prenatal testing (NIPT) for cellfree fetal DNA has emerged as a prenatal screening tool for the detection of aneuploidy in high-risk patients. In 2011 and 2012, Palomaki et al. ${ }^{4,5}$ published validation studies in 4,664 high-risk patients, reporting successful completion of massively parallel sequencing in $99.5 \%$ of samples, with sensitivities of 98.6, 100, and $100 \%$ for the detection of trisomies 21,18 and 13, respectively. In 2012, the MELISSA study group published data on the efficacy of massively parallel sequencing in 534 patients at high risk for trisomies 21,18 , and 13 , with sensitivities of 100 , 97.2 , and $78.6 \%$, respectively. The group reported a cumulative rate of $2.8 \%$ for "unclassified" results-now categorized as "aneuploidy suspected." An "unclassified" result was reported
Noninvasive prenatal testing had a combined sensitivity of $87.5 \%$ and specificity of $99.5 \%$ for detection of trisomies 21,18 , and 13 . There were "unclassified" results in $11.1 \%(5 / 45)$ of the patients. Over the study period, the number of patients requesting noninvasive prenatal testing increased monthly. The rate of amniocenteses significantly declined ( $8.1 \%$ before vs. $5.3 \%$ after noninvasive prenatal testing, $P<0.01)$.

Conclusion: An increase in uptake of noninvasive prenatal testing and a significant decline in amniocentesis procedures were observed. The rates of "unclassified," false-positive, and false-negative results were higher than anticipated based on published preclinical trials.

Genet Med advance online publication 27 March 2014

Key Words: aneuploidy screening; noninvasive prenatal testing

if the normalized chromosome value was within a borderline range where both false-positive and false-negative results were more likely to occur. In this article, these are referred to as "unclassified" results because this was the terminology used when the data were obtained.

Based on the emerging data supporting the utility of this technology, NIPT was offered at our institution as an option for prenatal aneuploidy screening in high-risk patients. ${ }^{7}$ The purpose of our study was to review our institution's experience with the use of NIPT for aneuploidy screening.

\section{MATERIALS AND METHODS}

A descriptive study of patients who received NIPT from January to September 2012 was performed. In January 2012, the Prenatal Diagnostics Unit at the University of North Carolina at Chapel Hill (UNC) began offering NIPT to patients at $\geq 10$ weeks gestational age with one or more of the following indications: advanced maternal age (AMA; $\geq 35$ years old at estimated date of delivery (EDD) with singleton or $\geq 32$ years old with dichorionic twin gestation), ultrasound findings suggestive of aneuploidy, family history of Down syndrome, or a positive first- or second-trimester serum screen (Figure 1). At our institution, all patients who meet the above criteria are offered genetic counseling to review the options of screening with the combined first-trimester screen (nuchal translucency, 


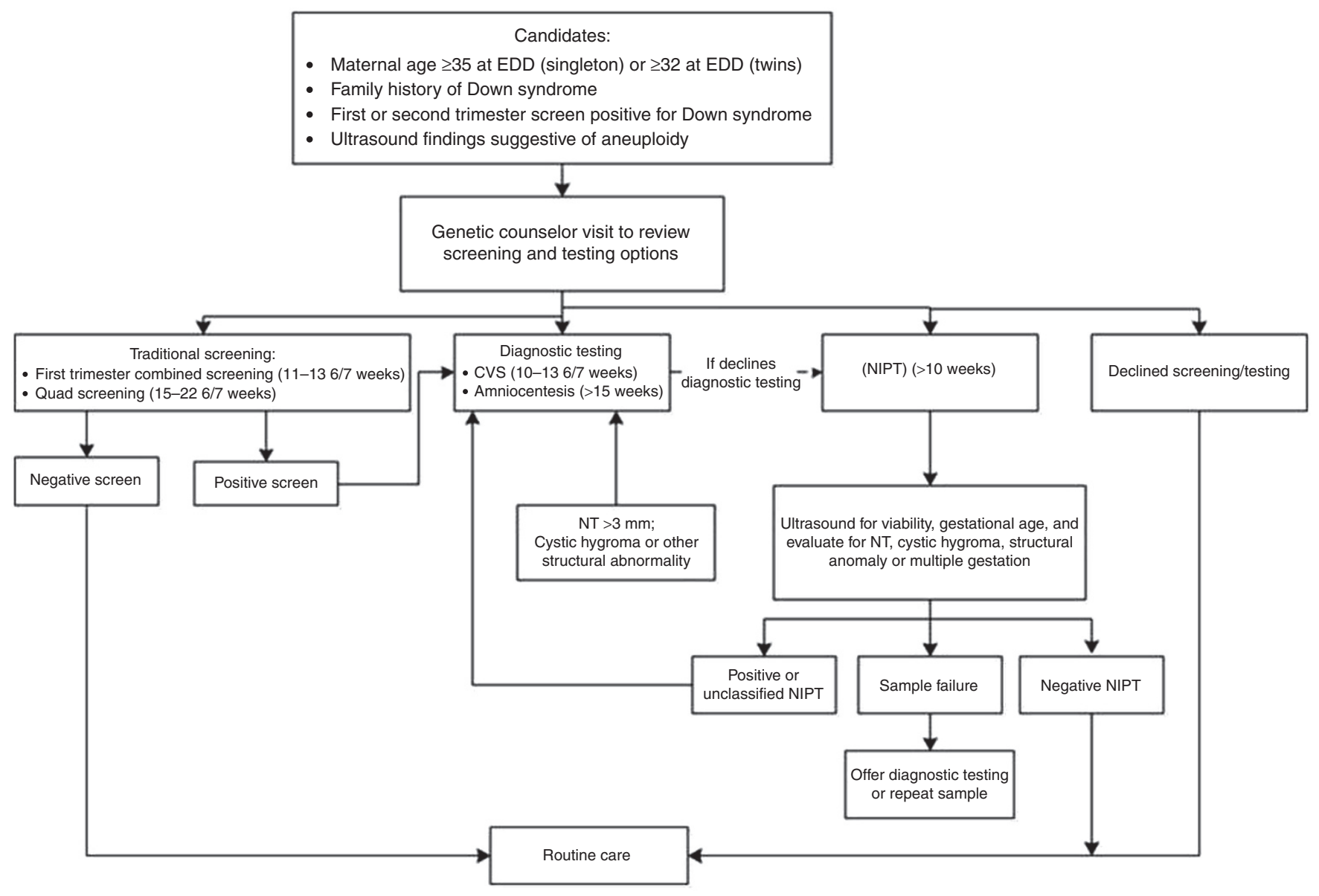

Figure 1 University of North Carolina at Chapel Hill (UNC) aneuploidy screening protocol. EDD, estimated date of delivery; NIPT, noninvasive prenatal testing; NT, nuchal translucency.

pregnancy-associated plasma protein $A$, and $\beta$-human chorionic gonadotropin), second-trimester quadruple screen (maternal serum alpha fetoprotein, $\beta$-human chorionic gonadotropin, estriol, and inhibin), or NIPT. Diagnostic testing options via CVS or amniocentesis are also offered. Although limited data have been published that validate the use of NIPT in twin gestations, ${ }^{8}$ testing was offered to these patients in select clinical situations. All patients electing NIPT underwent an ultrasound in order to confirm viability and gestational age and to evaluate for cystic hygroma, increased nuchal translucency, other structural anomalies, and multiple gestation.

Specimens collected were sent to either Sequenom Center for Molecular Medicine or Verinata Health. Multiple factors influenced the decision to use two laboratories during the study period. During this time, the Verinata laboratory reported "unclassified" results for samples with normalized chromosome values between 2.5 and 4.0. ${ }^{6}$ The normalized chromosome values were $>4.0$ for autosomal aneuploidy and $<2.5$ for unaffected fetuses. ${ }^{6}$ Invasive testing was recommended for patients with "unclassified" results due to the increased rate of aneuploidy $(28.6 \%(2 / 7))$ in the MELISSA study. ${ }^{6}$

Maternal demographics, prenatal aneuploidy testing results, and pregnancy outcomes were obtained from the patients' medical records. Body mass index $\left(\mathrm{kg} / \mathrm{m}^{2}\right)$ was calculated using the weight measured at the time of the screening test. We used the total number of genetic counseling visits to estimate the rates of screening and diagnostic testing in the 8 months before and after the availability of NIPT because genetic counseling is scheduled for all high-risk patients undergoing aneuploidy testing at our institution. Duplicate visits were removed to obtain accurate numbers. Univariate analysis was conducted using Student's $t$-test to compare continuous variables and $\chi^{2}$ or Fisher's exact test for categorical variables using Open Epi Version 2.3. The UNC Institutional Review Board approved this study.

\section{RESULTS}

During the study period, 208 patients underwent NIPT. The majority of patients were white $(62.9 \%)$ and of advanced maternal age (71.2\%) (Table 1). No significant differences in baseline demographics or indications for testing between those with normal and abnormal NIPT were observed (Table 1).

Over the study period, the number of NIPTs completed per month increased (Figure 2a). The rates of utilizing amniocentesis and all invasive procedures significantly declined after the availability of NIPT ( 8.1 before NIPT vs. $5.3 \%$ after NIPT, $P<0.01 ; 11.8$ before NIPT vs. $8.8 \%$ after NIPT, $P<0.01$, 
Table 1 Cohort demographics

\begin{tabular}{|c|c|c|c|c|}
\hline & All & $\begin{array}{c}\text { Normal } \\
\text { NIPT }^{a}\end{array}$ & $\begin{array}{c}\text { Abnormal } \\
\text { NIPT }^{\mathrm{a}}\end{array}$ & \\
\hline & $N=208$ & $n=192$ & $n=13$ & $P^{b}$ \\
\hline $\begin{array}{l}\text { Maternal age at EDD } \\
\text { (years) }\end{array}$ & & & & 0.53 \\
\hline Mean \pm SD & $36 \pm 5.5$ & $36 \pm 5.4$ & $37 \pm 7.4$ & \\
\hline Minimum-maximum & $19-47$ & $19-44$ & $20-47$ & \\
\hline Gestational age (weeks) & & & & 0.1 \\
\hline Mean \pm SD & $15.6 \pm 4.3$ & $15.5 \pm 4.1$ & $17.5 \pm 5.5$ & \\
\hline Minimum-maximum & $10-34$ & $10-34$ & $11.3-30.9$ & \\
\hline Trimester NIPT obtained & & & & 0.02 \\
\hline First & $111(53.4)$ & $103(53.7)$ & $5(38.5)$ & \\
\hline Second & $95(45.7)$ & $88(45.8)$ & $7(53.8)$ & \\
\hline Third & $2(1)$ & $1(0.5)$ & $1(1.1)$ & \\
\hline BMI $\left(\mathrm{kg} / \mathrm{m}^{2}\right)^{d}$ & & & & 0.83 \\
\hline Mean \pm SD & $27.7 \pm 6.8$ & $27.4 \pm 6.5$ & $27.8 \pm 6.3$ & \\
\hline Minimum-maximum & $16.8-51$ & $16.8-51$ & $20-42.3$ & \\
\hline Race & & & & 0.4 \\
\hline White & $131(62.9)$ & $123(64.1)$ & $6(46.2)$ & \\
\hline Black & $37(17.8)$ & $35(18.2)$ & $2(15.4)$ & \\
\hline Hispanic, nonwhite & $28(13.5)$ & $24(12.5)$ & $3(23.1)$ & \\
\hline Asian & $7(3.4)$ & $6(3.1)$ & $1(7.7)$ & \\
\hline Other & $5(2.4)$ & $4(2.1)$ & $1(7.7)$ & \\
\hline Laboratory & & & & $<0.01$ \\
\hline Sequenom & $163(78.4)$ & $156(81.3)$ & $4(30.8)$ & \\
\hline Verinata & $45(21.6)$ & $36(18.8)$ & $9(69.2)$ & \\
\hline Indication & & & & 0.94 \\
\hline AMA & $148(71.2)$ & $139(72.4)$ & $10(76.9)$ & \\
\hline AMA alone & 121 & 114 & 5 & \\
\hline $\begin{array}{l}\text { AMA + other } \\
\text { indication }^{\text {e }}\end{array}$ & 27 & 25 & 5 & \\
\hline Ultrasound abnormality & $26(12.5)$ & $23(12.0)$ & $2(15.4)$ & \\
\hline Abnormal serum screen & $29(13.9)$ & $25(13.0)$ & $1(7.7)$ & \\
\hline $\begin{array}{l}\text { First trimester (NT, } \\
\text { PAPP-A, } \beta-h C G \text { ) }\end{array}$ & 16 & 13 & 1 & \\
\hline $\begin{array}{l}\text { Quadruple (MSAFP, } \\
\text { hCG, estriol, inhibin) }\end{array}$ & 12 & 11 & 0 & \\
\hline Integrated & 1 & 1 & 0 & \\
\hline $\begin{array}{l}\text { Prior affected family } \\
\text { member }\end{array}$ & $3(1.4)$ & $3(1.6)$ & $0(0)$ & \\
\hline Other & $2(1.0)$ & $2(1.0)$ & $0(0)$ & \\
\hline $\begin{array}{l}\text { Twins (growth } \\
\text { discordance) }\end{array}$ & 1 & 1 & & \\
\hline Maternal anxiety & 1 & 1 & & \\
\hline
\end{tabular}

Data are $n$ (\% for column) unless otherwise specified.

AMA, advanced maternal age; BMI, body mass index; EDD, estimated date of delivery; $\mathrm{hCG}$, human chorionic gonadotropin; MSAFP, maternal serum alpha fetoprotein; NIPT, noninvasive prenatal testing; NT, nuchal translucency; PAPP-A, pregnancy-associated plasma protein A.

aExcludes three patients with insufficient fetal fraction/unreportable results.

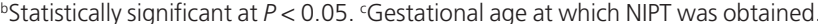
${ }^{\mathrm{D} D a t a}$ are missing for 30 patients. ${ }^{e}$ Other indications include ultrasound abnormality, abnormal serum screen, prior affected child, and/or twins. respectively) (Figure 2b). No significant change in the rates of CVS or first-trimester combined screens before and after NIPT availability was observed ( 3.3 vs. $3.7 \%, P=0.24 ; 46.7$ vs. $47.5 \%$, $P=0.31$, respectively) (Figure $2 \mathbf{b}$ ).

Aneuploidy was detected using NIPT in 3.8\% (8/208) of the cohort (Table 2). An "unclassified" result was reported in 11.1\% $(5 / 45)$ of samples analyzed at the laboratory that reported "unclassified" results (Table 2). Of the 13 patients with aneuploidy detected or "unclassified" results, 8 (61.5\%) underwent invasive prenatal diagnostic testing. No patient with a normal NIPT underwent invasive prenatal testing. Within our cohort, the sensitivity and the specificity were $87.5 \%$ and $99.5 \%$, respectively, for the detection of aneuploidy in chromosomes 21,18 , and 13. Aneuploidy was confirmed in seven patients, with six true positives for trisomies 21 and 18 and one false negative for trisomy 18 (Table 2). In addition, one false-positive result was observed for monosomy 18/trisomy 13. The fetus had a normal karyotype and microarray; however, metastatic maternal cancer was detected postpartum. This abnormal NIPT result was thought to be due to the multiple cytogenetic abnormalities found in the tumor. ${ }^{9}$ Of patients with "unclassified" NIPT results, one fetus had confirmed trisomy 18 and two had second-trimester fetal demises without postmortem genetic testing (Table 2). Two patients delivered term infants with normal newborn examinations (Table 2).

The NIPT result was not reportable because the fetal fraction was below the threshold in $1.4 \%(3 / 208)$ of the cohort. These patients had body mass indexes of 38.2, 44.6, and 47.3, and NIPT was performed at 11,10 , and 12 weeks, respectively. Only one of these three patients had repeat testing, and the fetal fraction remained below the threshold on repeat sample.

NIPT was performed in four sets of twins. Aneuploidy for chromosome 18 was detected in one set, subsequently resulting in the demise of one twin (Table 2). This fetus had phenotypic features consistent with trisomy 18 , including heart defect, clenched hands, and fetal growth restriction. The remainder of the twin sets (3/4) did not have aneuploidy detected on NIPT and had normal newborn examinations.

Delivery outcomes were available for 170 pregnancies (81.7\%). Thirty-eight patients delivered at outside institutions and therefore medical records were unavailable for review. The three patients with insufficient fetal fraction were excluded from further analysis. Of the 167 patients, 152 (91\%) had normal NIPT and normal newborn examinations. Among the remainder of patients with normal NIPT, three infants had genetic syndromes diagnosed on postnatal examinations, including 22q11.2 deletion syndrome, Vertebral anomalies, Anal atresia, Cardiac defects, Tracheo-Esophageal fistula, Renal anomalies, Limb abnormalities (VACTERL) association, and cerebroocularfacioskeletal syndrome/Cockayne syndrome with a microduplication of uncertain clinical significance on microarray. Two patients delivered nondysmorphic infants with structural anomalies (congenital pulmonary airway malformation and bilateral clubfeet). Three patients had fetal demises at 20, 22, and 36 weeks, and karyotype of the products of conception 
a

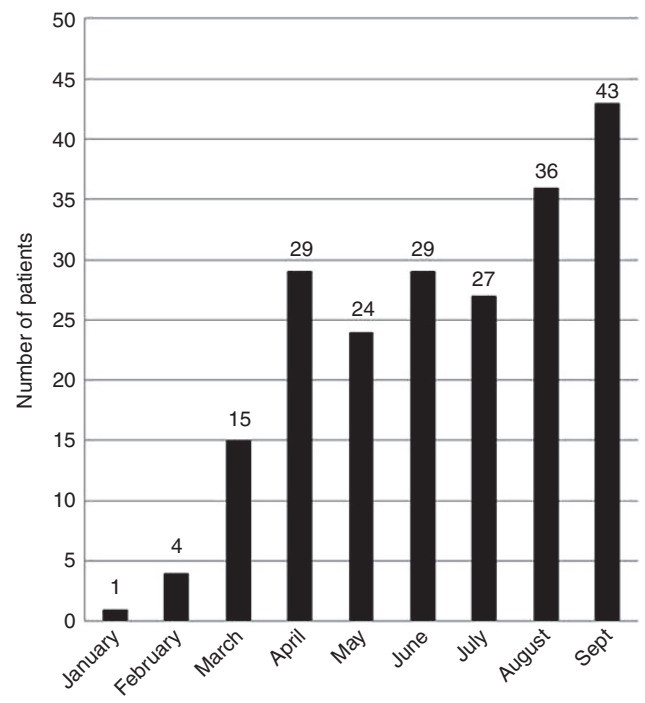

b

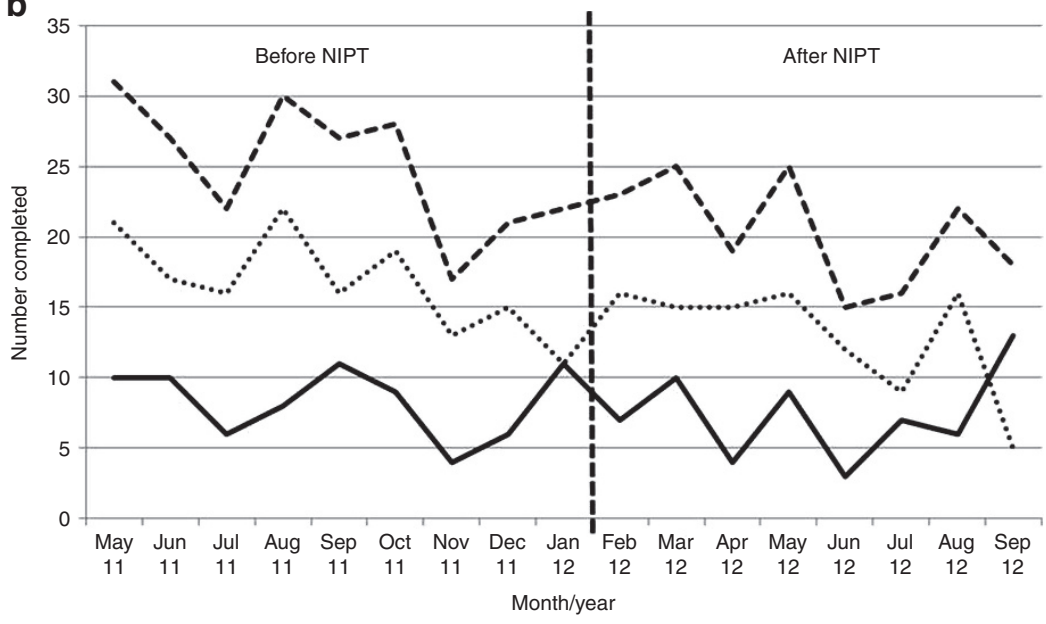

Rates before and after availability of NIPT (by number of genetic counseling visits over the time period) - - All invasive procedures: $11.8 \%(203 / 1,724)$ vs. $8.8 \%(185 / 2,156) ; P<0.01$ ...... Amniocentesis: $8.1 \%(139 / 1,724)$ vs. $5.3 \%(115 / 2,156) ; P<0.01$

- CVS: $3.7 \%(64 / 1,724)$ vs. $3.3 \%(70 / 2,156) ; P=0.24$

Figure 2 The number of patients who received noninvasive prenatal testing (NIPT) and invasive diagnostic procedures per month. (a) NIPT by month; (b) invasive procedures by month. CVS, chorionic villus sampling.

Table 2 Patients with abnormal NIPT

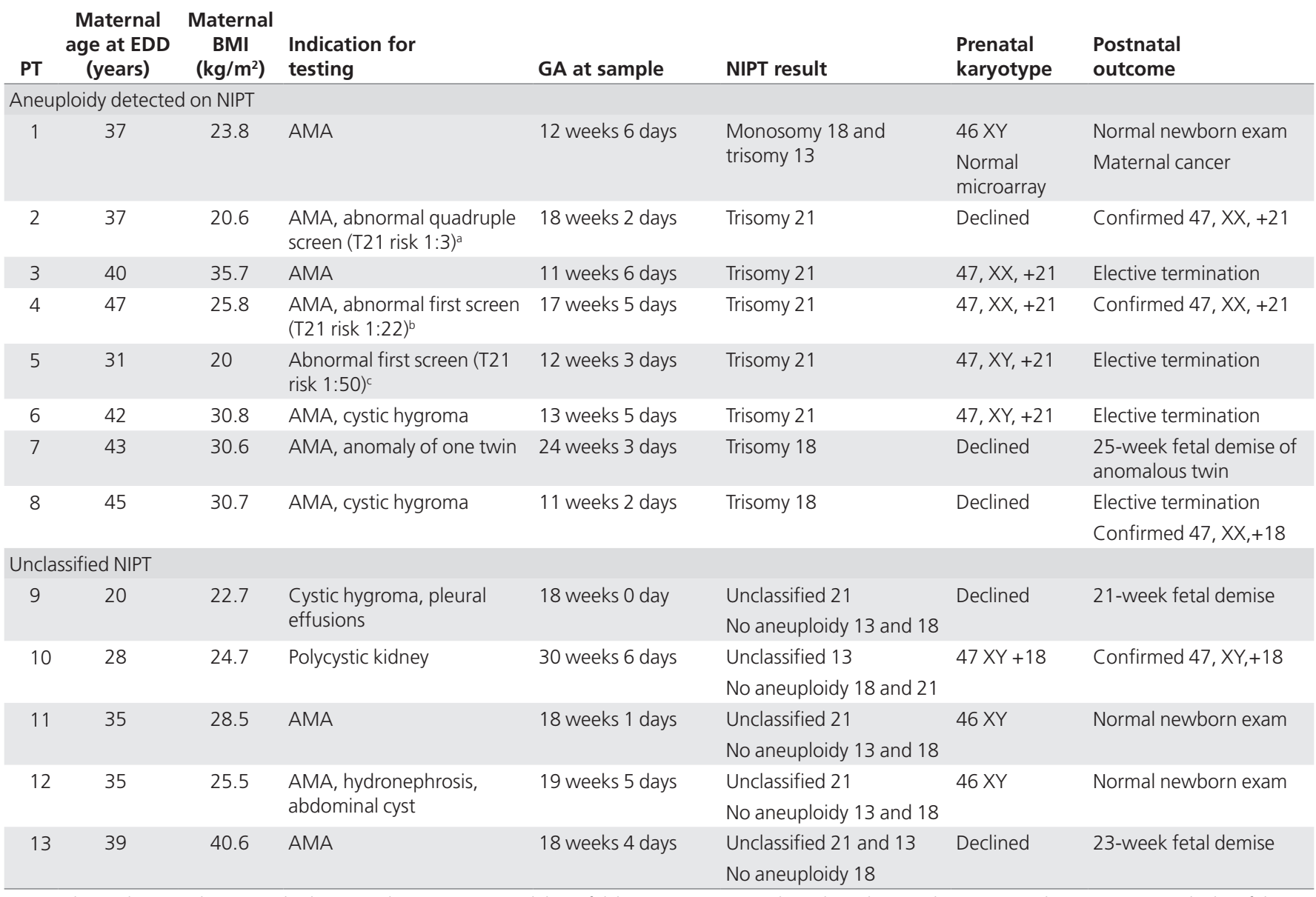

AMA, advanced maternal age; BMI, body mass index; EDD, estimated date of delivery; GA, gestational age; hCG, human chorionic gonadotropin; MoM, multiples of the median; MSAFP, maternal serum alpha fetoprotein; NIPT, noninvasive prenatal testing; NT, nuchal translucency; PAPP-A, pregnancy-associated plasma protein A; PT, patient; T, trisomy.

${ }^{a}$ Quadruple screen result: 1:3 risk for T21 (MSAFP 0.75 MoM, hCG 2.66 MoM, estriol 0.64 MoM, inhibin 3.27 MoM). bFirst-trimester screen result: 1:22 risk for T21 (NT $2.2 \mathrm{~mm}$, PAPP-A 0.49 MoM, $\beta$-hCG 1.06 MoM). cFirst-trimester screen result: 1:50 risk for T21 (NT 1.3 mm, PAPP-A 0.14 MoM, $\beta$-hCG 1.82 MoM). 
Table 3 Pregnancy outcomes

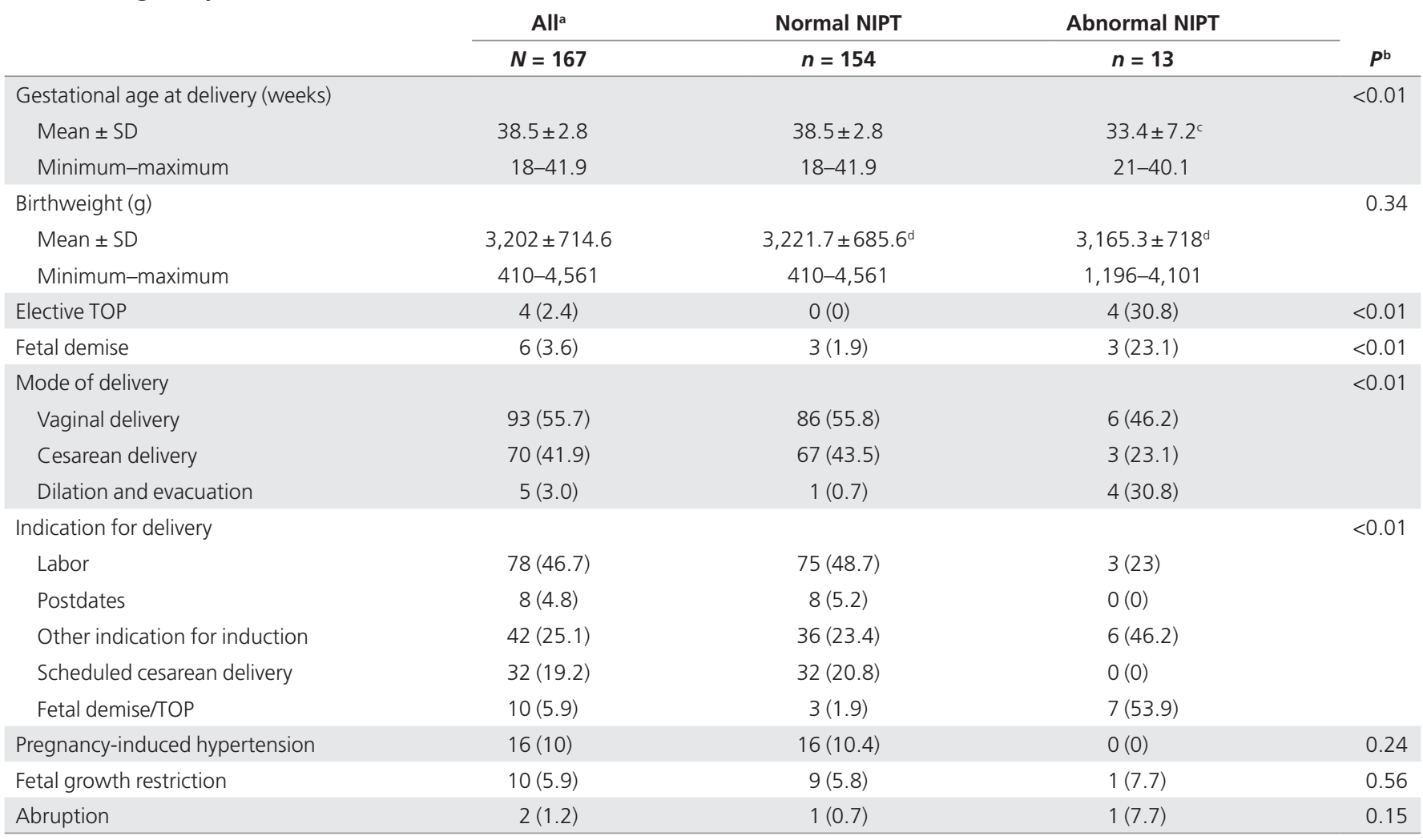

Data are $n$ (\% for column) unless otherwise specified.

NIPT, noninvasive prenatal testing; TOP, termination of pregnancy.

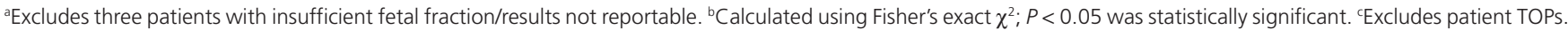
¿Birthweight not available for fetal demise/TOPs.

confirmed normal male karyotypes (46, XY) in two fetuses. The postnatal outcomes of the 13 patients with abnormal NIPT are available in Table 2.

The pregnancy outcomes for the cohort are available in Table 3. No significant differences were observed in pregnancyinduced hypertension, fetal growth restriction, or abruption in those with and without abnormal NIPT (Table 3). These outcomes were selected because they are thought to be placental mediated, and cell-free fetal DNA is thought to be primarily derived from apoptosis of placental cells.

\section{DISCUSSION}

Important factors in the decision to undergo NIPT include safety of the pregnancy, accuracy and earlier availability of results, and physician recommendations. ${ }^{10-12}$ The impact on total numbers of serum screens and invasive diagnostics tests is not yet known, but, over the study period, based on our single-institution experience, a steady increase in the number of patients undergoing NIPT, with a significant decline in the rates of amniocentesis and all invasive procedures, was observed. These findings are comparable to those obtained from the study of Chetty et al. ${ }^{13}$ in examining the uptake of NIPT.

The rates of first-trimester combined screens and CVS remained constant. We postulate two reasons for the lack of change in the rate of first-trimester screening. First, many women having first-trimester screening are not eligible for NIPT because they lack a risk factor for aneuploidy. Second, in our patient population, first-trimester screening is typically covered by insurance, whereas NIPT is not.

Multiple factors likely contributed to the stable rate of CVS in our cohort. Historically, the invasive diagnostic testing rate at our institution has been lower than in other parts of the country because the majority of patients of advanced maternal age with no other risk factors do not elect to undergo diagnostic testing. However, with increasing utilization of first-trimester screening and NIPT, many patients are now learning that their pregnancies are at high risk for a chromosome abnormality at a gestational age when CVS is available. Because procedure-related loss rates are comparable between CVS and second-trimester amniocentesis, ${ }^{7,14-17}$ many women who want definitive prenatal diagnosis may opt to have CVS in order to avoid the medical and psychological complications resulting from later prenatal diagnosis by amniocentesis.

Within our study population, NIPT appeared to have high sensitivity and specificity ( 87.5 and $99.5 \%$ ) for the detection of aneuploidy in chromosomes 21,18 , and 13 . One falsepositive result for monosomy 18/trisomy 13 in a patient with 
a subsequent postnatal diagnosis of maternal cancer was observed. ${ }^{9}$ There was also, one false negative for trisomy 18 in a patient with an "unclassified" result for trisomy 13 , but no aneuploidy was detected for trisomy 18 on NIPT. Mennuti et al. ${ }^{18}$ recently published eight cases with discordant results between NIPT results and cytogenetic testing of the pregnancy. Our combined experiences highlight the importance of confirming abnormal results with invasive testing. Although we strongly recommend patients confirm all abnormal NIPT results with prenatal diagnostic testing, this was not universally accepted, as only $61 \%$ of patients with an abnormal NIPT in our cohort received invasive testing. Those who declined confirmatory testing included a patient with a fetus with Down syndrome that delivered at term, an anomalous twin that subsequently resulted in a fetal demise, and a pregnancy termination in a patient with an ultrasound finding of cystic hygroma with postprocedure confirmation of trisomy 18.

In cases in which anomalies are detected by ultrasound, prenatal diagnostic testing via CVS or amniocentesis is recommended to obtain a sample for microarray analysis or karyotyping. ${ }^{19}$ However, NIPT is being increasingly used in clinical situations in which invasive testing is declined. In our study series, no patients with structural anomalies and normal NIPT chose invasive testing. However, two of these patients with normal NIPT and anomalies went on to deliver neonates diagnosed with genetic syndromes, 22q11.2 deletion syndrome and a rare single-gene disorder. This highlights the importance of reinforcing the fact that this technology screens for a limited number of aneuploidies and that patients and providers should not be falsely reassured by normal NIPT results in the setting of anomalies.

Also within our cohort, the overall rate of "unclassified" results was $11.1 \%$. This rate is higher than the cumulative "unclassified" rate of $2.8 \%$ as previously reported. ${ }^{6}$ We are unable to draw conclusions regarding whether the rate changed over time as, for various reasons, we discontinued using the laboratory that reported "unclassified" results. Although the exact reason for this higher "unclassified" rate in our cohort is unknown, we postulate that variations in the acquisition and handling of the specimens, such as phlebotomists, tube lot, or shipping conditions of the specimens, could theoretically have affected the samples. Poor pregnancy outcomes (two unexplained fetal demises and one karyotype-confirmed trisomy 18) occurred within this subset of patients with "unclassified" results. More information regarding "unclassified" results and adverse pregnancy outcomes is needed. Postnatal karyotypes should be obtained when NIPT results are "unclassified" or "aneuploidy suspected." Interestingly, all patients with "unclassified" results had samples drawn in the second and third trimester. Prior studies have demonstrated that fetal fraction increases with gestational age and is higher in cases of trisomy 21 as compared with euploid controls. ${ }^{20}$ However, fetal fraction is lower when the fetus has trisomy 18,13 , or monosomy $\mathrm{X}$, although it is still above the threshold fetal fraction of $4 \%$ that is needed to obtain a result. ${ }^{21}$ Therefore, we feel it is a coincidence, rather than an intrinsic problem of the test, that all our "unclassified" results occurred in the second and third trimester.

In three morbidly obese patients, the NIPT yielded no result, as there was an insufficient fetal fraction for analysis. In the MELISSA trial, the rate of insufficient fetal DNA detected was $3 \%$, but no differences in clinical features were noted after unblinding. ${ }^{6}$ Palomaki et al. ${ }^{4,5}$ found that $0.4 \%$ of samples had insufficient fetal fraction for analysis, and a strong negative association was noted between fetal fraction and maternal weight. Maternal obesity is associated with an increased amount of total cell-free DNA, resulting from adipocyte necrosis. ${ }^{22}$ Fetal fraction is the percentage of cell-free fetal DNA as compared with total cell-free DNA; thus, an increase in total cell-free DNA would result in a decreased fetal fraction. ${ }^{23}$ Prior studies have demonstrated that the fetal fraction is positively correlated with gestational age and negatively correlated with maternal weight. ${ }^{24,25}$ These data suggest that NIPT may not be as effective in obese women or may need to be completed at later gestational ages.

Because NIPT is able to routinely screen for trisomies 21,13 , and 18; sex-chromosome abnormalities; and low-prevalence microdeletion syndromes, it will be important to reassess the population-wide impact of routine NIPT. ${ }^{26,27}$ When NIPT is extended to a low-risk population in which the prevalence of the disease is low, the rate of false positives will increase. In addition, clinicians should also be mindful that this technology has the ability to detect underlying maternal conditions (i.e. maternal sex-chromosome abnormalities, mosaicism, and in our case, maternal cancer). ${ }^{9}$

Based on our initial experience, NIPT has had increasing uptake among patients who otherwise may have opted for other screening or diagnostic tests. Importantly, there was a significant decline in the rate of amniocenteses performed. The pitfalls with the implementation of this test are the higher "unclassified" rates and decreased success in obese patients. The occurrence of both false-positive and false-negatives results emphasizes that this technology is only a screening test and is not to be considered as replacement for amniocentesis or CVS in terms of diagnostic accuracy.

\section{DISCLOSURE}

The authors declare no conflict of interest.

\section{REFERENCES}

1. Screening for fetal chromosomal abnormalities. ACOG Practice Bulletin No. 77. American College of Obstetricians and Gynecologists. Obstet Gynecol 2007;109:217-227.

2. Hill M, Barrett AN, White $H$, Chitty LS. Uses of cell free fetal DNA in maternal circulation. Best Pract Res Clin Obstet Gynaeco/ 2012;26:639-654

3. Ehrich M, Deciu C, Zwiefelhofer T, et al. Noninvasive detection of fetal trisomy 21 by sequencing of DNA in maternal blood: a study in a clinical setting. Am J Obstet Gynecol 2011;204:205 e201-211.

4. Palomaki GE, Kloza EM, Lambert-Messerlian GM, et al. DNA sequencing of maternal plasma to detect Down syndrome: an international clinical validation study. Genet Med 2011;13:913-920.

5. Palomaki GE, Deciu C, Kloza EM, et al. DNA sequencing of maternal plasma reliably identifies trisomy 18 and trisomy 13 as well as Down syndrome: an international collaborative study. Genet Med 2012;14:296-305. 
6. Bianchi DW, Platt LD, Goldberg JD, Abuhamad AZ, Sehnert AJ, Rava RP MatErnal BLood IS Source to Accurately diagnose fetal aneuploidy (MELISSA) Study Group. Genome-wide fetal aneuploidy detection by maternal plasma DNA sequencing. Obstet Gynecol 2012;119:890-901.

7. Noninvasive prenatal testing for fetal aneuploidy. American College of Obstetricians and Gynecologists. Committee Committee Opinion No. 545. Obstet Gyneco/ 2012;120:1532-1534

8. Canick JA, Kloza EM, Lambert-Messerlian GM, et al. DNA sequencing of maternal plasma to identify Down syndrome and other trisomies in multiple gestations. Prenat Diagn 2012;32:730-734.

9. Osborne CM, Hardisty E, Devers P, et al. Discordant noninvasive prenatal testing results in a patient subsequently diagnosed with metastatic disease. Prenat Diagn 2013;33:609-611.

10. Tischler R, Hudgins L, Blumenfeld YJ, Greely HT, Ormond KE. Noninvasive prenatal diagnosis: pregnant women's interest and expected uptake. Prenat Diagn 2011;31:1292-1299

11. Hill M, Fisher J, Chitty LS, Morris S. Women's and health professionals preferences for prenatal tests for Down syndrome: a discrete choice experiment to contrast noninvasive prenatal diagnosis with current invasive tests. Genet Med 2012;14:905-913.

12. Susman MR, Amor DJ, Muggli E, Jaques AM, Halliday J. Using population-based data to predict the impact of introducing noninvasive prenatal diagnosis for Down syndrome. Genet Med 2010;12:298-303.

13. Chetty S, Garabedian MJ, Norton ME. Uptake of noninvasive prenatal testing (NIPT) in women following positive aneuploidy screening. Prenat Diagn 2013;33:542-546.

14. Jackson LG, Zachary JM, Fowler SE, et al. A randomized comparison of transcervical and transabdominal chorionic-villus sampling. The U.S. National Institute of Child Health and Human Development ChorionicVillus Sampling and Amniocentesis Study Group. N Engl J Med 1992;327: 594-598.

15. Rhoads GG, Jackson LG, Schlesselman SE, et al. The safety and efficacy of chorionic villus sampling for early prenatal diagnosis of cytogenetic abnormalities. N Engl J Med 1989;320:609-617.
16. Multicentre randomised clinical trial of chorion villus sampling and amniocentesis. First report. Canadian Collaborative CVS-Amniocentesis Clinical Trial Group. Lancet 1989:1:1-6.

17. Caughey AB, Hopkins LM, Norton ME. Chorionic villus sampling compared with amniocentesis and the difference in the rate of pregnancy loss. Obstet Gynecol 2006;108(3 Pt 1):612-616.

18. Mennuti MT, Cherry AM, Morrissette JJ, Dugoff L. Is it time to sound an alarm about false-positive cell-free DNA testing for fetal aneuploidy? Am J Obstet Gynecol 2013;209:415-419.

19. The use of chromosomal microarray analysis in prenatal diagnosis. Committee Opinion. No. 581. Obstet Gynecol 2013;122:1374-1377.

20. Lee T, LeShane ES, Messerlian GM, et al. Down syndrome and cell-free fetal DNA in archived maternal serum. Am J Obstet Gynecol 2002;187:1217-1221.

21. Rava RP, Srinivasan A, Sehnert AJ, Bianchi DW. Circulating fetal cell-free DNA fractions differ in autosomal aneuploidies and monosomy X. Clin Chem 2013;60:1-8

22. Haghiac M, Vora NL, Basu S, et al. Increased death of adipose cells, a path to release cell-free DNA into systemic circulation of obese women. Obesity (Silver Spring) 2012;20:2213-2219.

23. Vora NL, Johnson KL, Basu S, Catalano PM, Hauguel-De Mouzon S, Bianchi DW. A multifactorial relationship exists between total circulating cell-free DNA levels and maternal BMI. Prenat Diagn 2012;32:912-914

24. Ashoor G, Syngelaki A, Poon LC, Rezende JC, Nicolaides KH. Fetal fraction in maternal plasma cell-free DNA at 11-13 weeks' gestation: relation to maternal and fetal characteristics. Ultrasound Obstet Gynecol 2013;41:26-32.

25. Wang E, Batey A, Struble C, Musci T, Song K, Oliphant A. Gestational age and maternal weight effects on fetal cell-free DNA in maternal plasma. Prenat Diagn 2013;33:662-666

26. Srinivasan A, Bianchi DW, Huang H, Sehnert AJ, Rava RP. Noninvasive detection of fetal subchromosome abnormalities via deep sequencing of maternal plasma. Am J Hum Genet 2013:92:167-176.

27. Jensen TJ, Dzakula Z, Deciu C, van den Boom D, Ehrich M. Detection of microdeletion 22 q11.2 in a fetus by next-generation sequencing of maternal plasma. Clin Chem 2012;58:1148-1151. 